\title{
Rounding Frequency and Hospital Length of Stay for Children With Respiratory Illnesses: A Simulation Study
}

\author{
Joseph East, BS ${ }^{1,2}$, Allison Cator, MD, PhD, Emily Burns', Tara Lynn O'Gara, BSE ${ }^{1}$, Jason Card, BSE ${ }^{1}$, \\ Amy Cohn, $\mathrm{PhD}^{1,2,4}$, Michelle Macy, MD, MS ${ }^{3,5}$
}

\begin{abstract}
${ }^{1}$ Industrial and Operations Engineering Department, University of Michigan, Ann Arbor, Michigan; ${ }^{2 H e a l t h ~ M a n a g e m e n t ~ a n d ~ P o l i c y ~ D e p a r t m e n t, ~}$ School of Public Health, University of Michigan, Ann Arbor, Michigan; ${ }^{3}$ Children's Emergency Services, Department of Emergency Medicine, University of Michigan, Ann Arbor, Michigan; ${ }^{4}$ Center for Healthcare Engineering and Patient Safety, University of Michigan, Ann Arbor, Michigan; ${ }^{5}$ Child Health Evaluation and Research Unit, University of Michigan, Ann Arbor, Michigan.
\end{abstract}

BACKGROUND: Patient discharge from the hospital is linked to physician-led rounds, whereas discharge from the emergency department (ED) is more fluid. The relationship between rounding and length of stay (LOS) has not been quantitatively described.

OBJECTIVES: To describe the arrival and discharge patterns in the ED and inpatient settings for children with respiratory illnesses and to explore how the timing and frequency of rounding could impact LOS.

DESIGN/SETTING: Retrospective administrative data analyses of visits for respiratory illnesses to a tertiary care pediatric ED from May 2007 to April 2010.

METHODS: ED visits for common respiratory conditions were selected based on International Classification of Diseases, 9th Revision, Clinical Modification codes, excluding complex comorbid conditions, severe illness, and intensive care unit admission. Discharge time was plotted against arrival time for the ED and inpatient unit. LOS was calcu- lated. A Monte Carlo simulation model was developed to explore the influence of additional rounds on inpatient LOS.

RESULTS: Of the 5503 included visits, 1285 (23.4\%) resulted in inpatient care. Discharges from the ED typically occurred 2 to 5 hours after arrival, whereas most inpatient discharges occurred between 11 AM and 6 PM regardless of admission time. Simulating 1 additional rounding session decreased predicted inpatient LOS by approximately 5 hours.

CONCLUSIONS: In contrast to ED discharges that occurred around the clock, inpatient discharges for children with respiratory illnesses were concentrated during afternoon hours. Increasing rounding frequency may improve hospital efficiency but could result in unintended consequences such as fewer opportunities for patient education. Journal of Hospital Medicine 2013;8:678-683. (c) 2013 Society of Hospital Medicine
Hospitals are facing growing pressures to operate more efficiently, spurring interest in improving patient flow from the emergency department (ED) to inpatient unit to home. Children's hospitals are often at high occupancy, ${ }^{1}$ and EDs are increasingly an entry point for hospital admission. ${ }^{2,3}$

Among children who require brief episodes of hospital-based care, hospital processes, especially those associated with discharge, can greatly impact length of stay (LOS). Patients ready for discharge from inpatient units are typically identified through formal physician-led rounds, in contrast to EDs where discharges occur on a more continual basis. Quantitative descriptions of rounding frequency and LOS are lacking.

\footnotetext{
*Address for correspondence and reprint requests: Allison Cator, MD, Department of Emergency Medicine, University of Michigan, $1500 \mathrm{E}$. Medical Center Dr., TC B1-380, Ann Arbor, Ml 48109; Telephone: 734232-6166; Fax: 734-763-9298; E-mail: acator@med.umich.edu

Additional Supporting Information may be found in the online version of this article.

Received: July 25, 2013; Revised: September 27, 2013; Accepted: September 27, 2013

2013 Society of Hospital Medicine DOI 10.1002/jhm.2097

Published online in Wiley Online Library (Wileyonlinelibrary.com).
}

The focus of this study was the population of children who had visits for select respiratory illnesses (e.g., asthma, bronchiolitis, pneumonia, and croup) for which there is general consensus regarding treatment, admission, and discharge criteria. ${ }^{4-7}$ The selected illnesses represent common reasons for ED visits $^{8}$ and hospitalizations. ${ }^{9}$ Hospital stays for these conditions tend to be brief, often 1 to 2 days in duration, ${ }^{10}$ and repeated assessments are necessary to determine suitability for discharge.

The primary objectives of this study were to compare discharge patterns in the ED and inpatient settings and to quantify the relationship between discharge timing and LOS in these different clinical settings. A simulation was then used to predict the effect of the timing and frequency of physician-led rounds on hospital LOS. We hypothesized that increased frequency of simulated physician-led rounds would lead to meaningful reductions in predicted hospital LOS for children admitted from the ED.

\section{METHODS}

Retrospective analyses were conducted using hospital administrative data from pediatric ED visits and resultant inpatient stays. The University of Michigan Institutional Review Board approved the study. 


\section{Setting}

C. S. Mott Children's Hospital at the University of Michigan is a suburban academic, tertiary care hospital located in Ann Arbor, Michigan. The pediatric emergency department had approximately 20,000 visits per year during the study period, and children were treated in an 11-bed area. There were 106 pediatric inpatient general care beds during the study years.

\section{Visit Selection}

ED visits made by children $<18$ years old during the 3-year period between May 1, 2007 and April 30, 2010 were considered eligible for study. Visits were included in the study based on the presence of International Classification of Disease, 9th Revision, Clinical Modification (ICD-9-CM) codes for respiratory conditions (asthma [493.xx], pneumonia [480.x486.x], croup [464.4], bronchiolitis [466.x], acute bronchospasm [519.11], wheezing [786.07]) in the top 3 ED diagnoses. Visits were excluded if the hospital discharge diagnoses included ICD-9-CM codes determined a priori by the study team to represent severe illness (e.g., respiratory failure) or complicated medical conditions (e.g., ventilator dependence) unlikely to respond to short stay care (see Supporting Appendices 1 through 3 in the online version of this article). ED visits that resulted in an admission to the intensive care unit (ICU), a procedure, or telemetry were excluded because these children would be unlikely to respond to short stay care.

\section{Variables}

Subject demographic characteristics included age, gender, race, and payer. Visit-related variables included date and time of ED arrival, date and time of ED disposition (to home or admission), and date and time of hospital discharge if admitted. Arrival date and time were used to determine season (winter: DecemberMarch, spring/summer: April-August, fall: SeptemberNovember) and shift (day: 8 AM-4 PM, evening: 4 PMmidnight, night: midnight-8 AM).

\section{Outcome}

LOS was the primary outcome. ED LOS was calculated by subtracting the date and time of ED arrival from the date and time of ED disposition. Inpatient LOS was calculated by subtracting the date and time of arrival to the inpatient unit from the date and time of discharge from the hospital admission, discharge, transfer (ADT) system. Total LOS was calculated by subtracting the date and time of ED arrival from the date and time of discharge from either the ED or hospital as appropriate.

\section{Analyses}

Descriptive statistics were calculated for patient demographics and actual LOS. To describe discharge timing patterns in the ED and inpatient unit, $24 \times 24$, 576-cell matrices were produced with arrival hour along a horizontal axis and discharge hour along a vertical axis. Visits were grouped by hour of ED or inpatient arrival and the number of these visits that were discharged in each hour was plotted in the appropriate cell in the matrix. Cells were then shaded according to the proportion of discharges that occurred at that hour, for each hour of arrival.

Finally, Monte Carlo simulations were designed to illustrate the impact of the timing and frequency of rounds on inpatient LOS. Historical arrival rates were utilized in the simulations. Because medication administration times were not available in the dataset, active treatment times were fixed at 12 hours and 20 hours. The fixed treatment time of 12 hours was based on research by McConnochie et al., ${ }^{11}$ which found that roughly $65 \%$ of children hospitalized for asthma received frequent nebulized treatments for 16 hours (two 8-hour nursing shifts). Four hours were subtracted from this treatment time because our sample included ED treatment times, and local experience demonstrates that children receive at least 4 hours of ED treatment prior to transfer to an inpatient bed. The 20-hour fixed treatment time was selected to simulate a sicker population of children.

We assumed that physician-led rounds were the decision point leading to hospital discharge, and that, at minimum, rounds occurred once each morning. The simulation began with 1 physician-led rounding session occurring at 9 AM. Rounding sessions were then added to the model up to a maximum of 24 hourly rounds. Rounding every hour was considered to be analogous to the 24-hour operation of an ED, in which patients are discharged at all hours. A 4-hour lag time between the start of first morning rounds and patient discharge was assumed based on historical discharge times. The lag time for additional rounding times was varied, from a minimum of 2 hours for afternoon rounds to a maximum of 10 hours for evening rounds to allow the patient to sleep. Each simulation completed 10,000 iterations and $95 \%$ confidence intervals (CIs) were calculated. All analyses were conducted in Excel 2010 (Microsoft Corp., Redmond, WA).

\section{RESULTS \\ Study Sample Characteristics}

Of the 57,639 pediatric ED visits during the 3-year study period, 5699 were diagnosed with respiratory conditions considered eligible for this study, and 5503 $(96.6 \%)$ were included in analyses. Hospitalization was the outcome for 1285 (23.4\%) ED visits. Demographic characteristics of the study sample are presented in Table 1. Almost half of the visits were by children $<3$ years old. Visits for respiratory conditions peaked in the fall and winter months. Daily peaks in ED arrivals occurred in the late afternoon. 


\section{Length of Stay}

For visits meeting study criteria, median ED LOS was 3.0 hours (interquartile range [IQR] 2.1-4.1) among ED discharges and 5.1 hours (IQR 4.0-6.6) among admissions. Of the inpatients, $8.4 \%$ were admitted

\begin{tabular}{|c|c|c|}
\hline Characteristic & $\mathrm{n}$ & $\%$ \\
\hline Male & 3291 & 59.8 \\
\hline \multicolumn{3}{|l|}{ Age } \\
\hline $0-2$ years & 2716 & 49.4 \\
\hline $3-5$ years & 1305 & 23.7 \\
\hline $6-12$ years & 1128 & 20.5 \\
\hline$>12$ years & 354 & 6.4 \\
\hline \multicolumn{3}{|l|}{ Season at presentation } \\
\hline Fall (September-November) & 1779 & 32.3 \\
\hline Winter (December-March) & 2429 & 44.1 \\
\hline Spring/Summer (April-August) & 1295 & 23.5 \\
\hline \multicolumn{3}{|l|}{ Shift at time of ED arrival } \\
\hline Day (8 AM-4 PM) & 1939 & 35.2 \\
\hline Evening (4 PM-12 AM) & 2296 & 41.7 \\
\hline Overnight (12 AM -8 AM) & 1268 & 23.0 \\
\hline \multicolumn{3}{|l|}{ Payer type } \\
\hline Public & 1996 & 36.3 \\
\hline Private & 3385 & 61.5 \\
\hline Other & 122 & 2.2 \\
\hline Admitted to general care unit & 1285 & 23.4 \\
\hline \multicolumn{3}{|l|}{ ICD-9 diagnoses } \\
\hline Asthma (493.xx) & 2229 & 40.5 \\
\hline Pneumonia (480.x-486.x) & 1006 & 18.3 \\
\hline Croup (464.4) & 1048 & 19.0 \\
\hline Bronchiolitis (466.x) & 605 & 11.0 \\
\hline Other $(519.11,786.07$, and multiple diagnoses $)$ & 615 & 11.2 \\
\hline
\end{tabular}

NOTE: Abbreviations: ED, emergency department; ICD-9, International Classification of Diseases, 9th Revision. and discharged on the same day, $37.3 \%$ were admitted for 1 night, $27.0 \%$ were admitted for 2 nights, and $27.3 \%$ were admitted for 3 or more nights. Median inpatient LOS was 41.2 hours (IQR 23.466.4), with median total LOS of 46.7 hours (IQR 29.3-71.5).

\section{Arrival and Discharge Patterns}

Figure 1 illustrates the relationship between the ED arrival and disposition times for all included visits. Figure 2 illustrates the relationship between the inpatient arrival and discharge times among the subset of visits that received inpatient care. Children with respiratory illness arrived to the ED at all hours of the day and night and generally were discharged 2 to 5 hours after arrival regardless of the time of day. In contrast, children admitted to the inpatient setting were most commonly discharged between $11 \mathrm{AM}$ and $6 \mathrm{PM}$, regardless of the time of admission.

\section{Simulation}

The influence of timing and frequency of rounds on inpatient LOS is shown in Table 2. Once-daily rounds resulted in a predicted LOS of 27.24 hours $(95 \%$ CI: 27.07-27.41) for the 12-hour active treatment time simulation and 36.93 hours (95\% CI: 36.70-37.16) for the 20-hour active treatment time. There were incremental reductions in predicted LOS with each additional rounding session. When the number of rounding sessions reached 24, predicted LOS was 14.5 hours (95\% CI: 14.49-14.51) for the 12-hour active treatment time simulation and 22.5 hours $(95 \%$ CI: 22.49-22.51) for the 20-hour active treatment time.

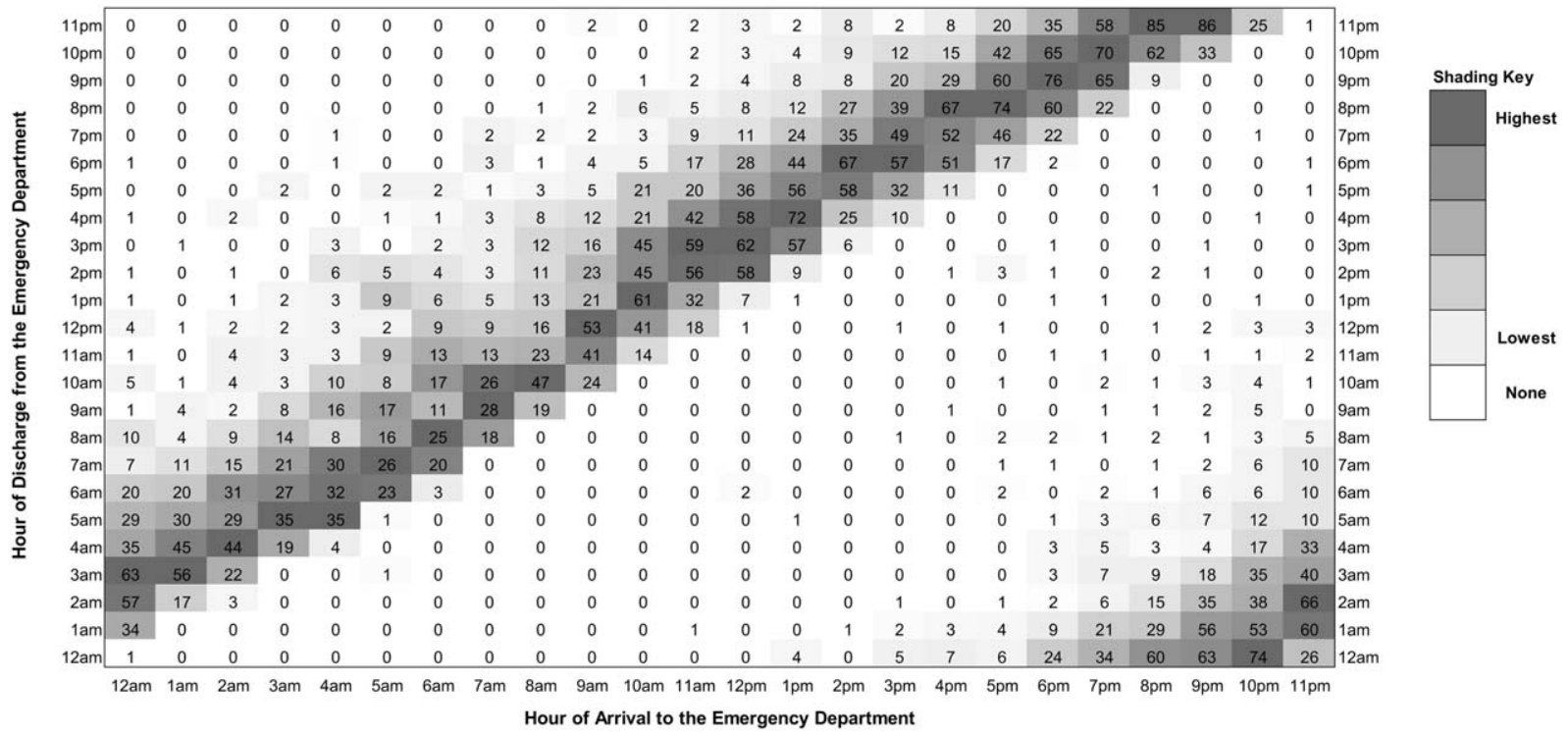

FIG. 1. Arrival versus discharge hour for respiratory patients in the emergency department. Each cell in the matrix indicates the number of discharges that occurred at a given hour (rows) for each hour of arrival (columns). Within each column the values total the number of arrivals for each hour of the day. Cells were shaded according to the relative proportion of visits that were discharged at a given hour within the group of visits with the same arrival hour. The darkest gray shading indicates the highest proportion of discharges for that arrival hour; the lightest gray shading indicates the lowest proportion of discharges for that arrival hour. Cells that have no discharges for an arrival hour do not have any shading. 


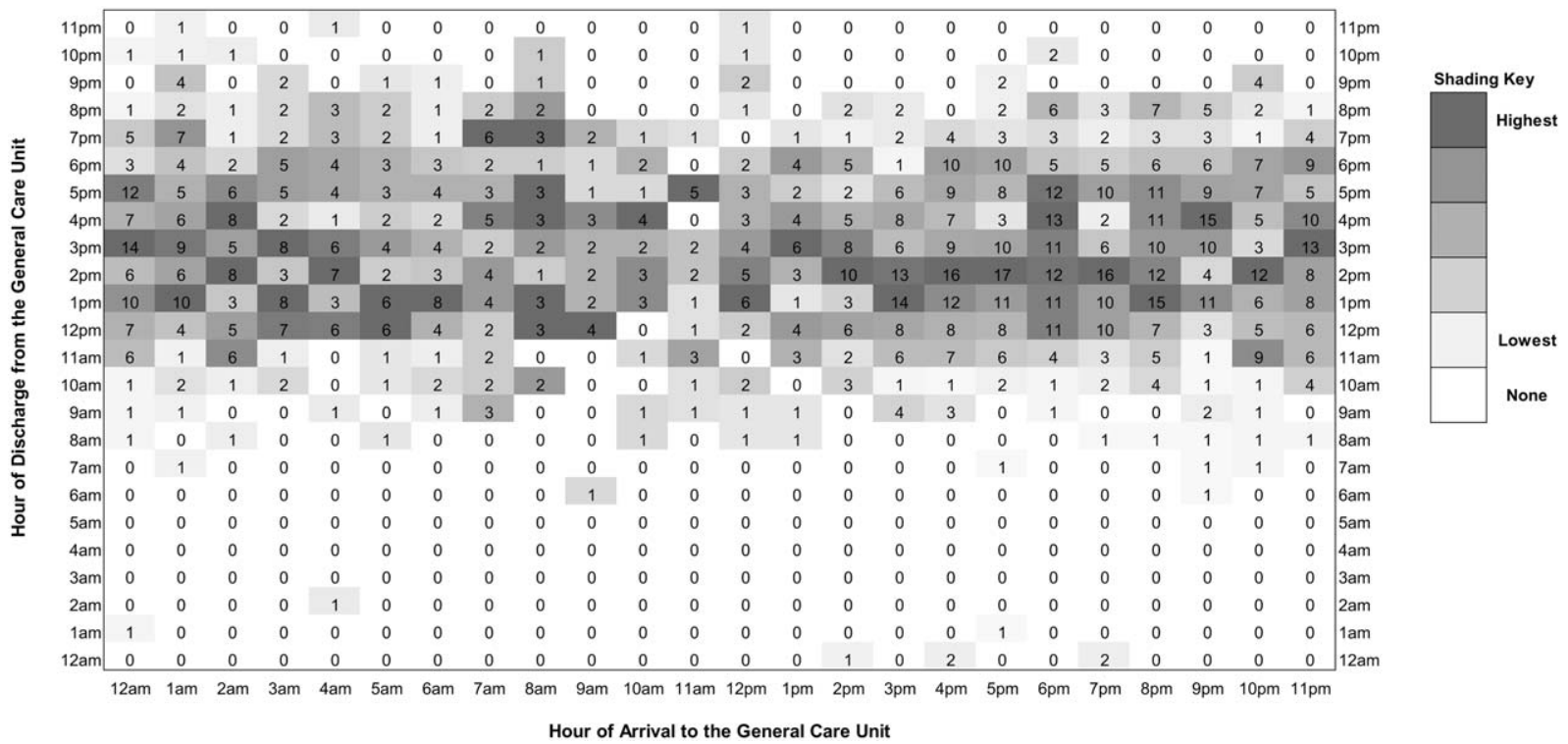

FIG. 2. Arrival versus discharge hour for respiratory patients in the general care unit. Each cell in the matrix indicates the number of discharges that occurred at a given hour (rows) for each hour of arrival (columns). Within each column the values total the number of arrivals for each hour of the day. Cells were shaded according to the relative proportion of visits that were discharged at a given hour within the group of visits with the same arrival hour. The darkest gray shading indicates the highest proportion of discharges for that arrival hour; the lightest gray shading indicates the lowest proportion of discharges for that arrival hour. Cells that have no discharges for an arrival hour do not have any shading.

TABLE 2. Monte Carlo Simulation Results in Terms of Change in Length of Stay

\begin{tabular}{|c|c|c|c|c|c|c|}
\hline \multirow[b]{2}{*}{$\begin{array}{l}\text { No. of } \\
\text { Rounds }\end{array}$} & \multirow[b]{2}{*}{ Time of Rounds } & \multirow[b]{2}{*}{$\begin{array}{c}\text { Rounds to } \\
\text { Discharge (Hours) }\end{array}$} & \multicolumn{2}{|c|}{ 12-Hour Active Treatment Time } & \multicolumn{2}{|c|}{ 20-Hour Active Treatment Time } \\
\hline & & & $\begin{array}{l}\text { Change in Length } \\
\text { of Stay (Hours) }\end{array}$ & $\begin{array}{l}\% \text { of Reference } \\
\text { Length of Stay }\end{array}$ & $\begin{array}{l}\text { Change in Length of } \\
\text { Stay (Hours) }\end{array}$ & $\begin{array}{l}\% \text { of Reference } \\
\text { Length of Stay }\end{array}$ \\
\hline 1 & 0900 & 4 & 27.24 (reference) & & 36.93 (reference) & \\
\hline 2 & $\begin{array}{l}0900 \\
1500\end{array}$ & $\begin{array}{l}4 \\
4\end{array}$ & -4.84 & $17.8 \%$ & -5.34 & $14.4 \%$ \\
\hline 2 & $\begin{array}{l}0900 \\
1500\end{array}$ & $\begin{array}{l}4 \\
2\end{array}$ & -5.31 & $19.5 \%$ & -5.90 & $16.0 \%$ \\
\hline 3 & $\begin{array}{l}0900 \\
1500 \\
2100\end{array}$ & $\begin{array}{c}4 \\
2 \\
10\end{array}$ & -6.24 & $22.9 \%$ & -7.94 & $21.5 \%$ \\
\hline 3 & $\begin{array}{l}0900 \\
1500 \\
2100\end{array}$ & $\begin{array}{l}4 \\
2 \\
2\end{array}$ & -7.32 & $26.9 \%$ & -10.49 & $28.4 \%$ \\
\hline 24 & Hourly & 2 & -12.74 & $46.8 \%$ & -14.2 & $38.7 \%$ \\
\hline
\end{tabular}

\section{DISCUSSION}

Our study illustrates dramatic differences in the discharge patterns from the ED and inpatient settings for children with respiratory illnesses. Although discharges from the ED occur at all hours, inpatient discharges were concentrated during midday. The time of discharge from the ED was highly related to the time of arrival, implying that any lag between discharge decision making and discharge was independent of time of arrival. The absence of a clear relationship between inpatient discharges and arrivals suggests that factors other than the clinical status of patients affect hospital LOS.

Because physician-led rounds have traditionally been the point of decision-making regarding inpatient discharge readiness, we hypothesized that increasing the frequency of rounds could reduce LOS. Within the parameters set in our simulation models, our results support this hypothesis. As the number of rounding sessions increased, hospital LOS decreased and the amount of time a child waited to be identified as ready for discharge approached zero. Simulating hourly rounding, analogous to the ED setting, resulted in the greatest reductions in inpatient LOS. Our findings have important implications for hospital operations and discharge policies given that children with respiratory conditions commonly experience short stay hospitalizations, ${ }^{12,13}$ but few children's hospitals have put in place models of care that differentiate the needs of short stay patients from those of inpatients requiring longer LOS. ${ }^{14}$ To operationalize inpatient discharges as soon as a child is well enough to go 
home, parental expectations for discharge timing would need to be set, and discharge planning would need to begin at the start of each hospital stay.

Although there have been numerous studies on ED crowding, its causes, and potential solutions, ${ }^{15-20}$ this study is the first to our knowledge to demonstrate differences in discharge timing between the ED and inpatient general care unit. High levels of hospital occupancy decrease patient flow in both ED and inpatient units. ${ }^{21-25}$ Shifting inpatient discharge for adult patients to earlier in the day can reduce or eliminate inpatient boarding in the ED. ${ }^{26,27}$ Evaluation of these relationships in pediatric populations are needed because of the unique care requirements of acutely ill children who often respond rapidly to hospital-based treatment. In a recent opinion piece, Iantorno and Fieldston discourage hospitals from setting specified time targets for discharges and propose that highquality care includes afternoon and evening discharges. ${ }^{28}$ The optimal timing for hospital discharges has not yet been defined, but our results indicate there is potential to reduce excess time spent in hospitals through the addition of rounds that would identify discharges throughout the day.

A simulation study based on hospital administrative data cannot determine a causal relationship between physician-led rounds and hospital LOS. Still, our findings can generate discussions about hospital discharge policies and patient throughput initiatives. Hospitals can simulate other approaches that may apply to their institutional operations, such as changing rounding processes or adding an observation unit where discharges occur after hours. Hospitals could then pilot approaches locally. With the potential for unintended consequences of patient throughput initiatives that focus only on LOS as an outcome, pilot programs should be designed to track not only ED and inpatient LOS but also family satisfaction, access to follow-up visits in primary care, need for reassessment in urgent care centers and EDs, and hospital readmissions.

The feasibility and acceptability of afternoon and evening rounds to improve patient throughput must be considered. Clinicians caring for hospitalized patients may have competing demands on their time, such as other clinical obligations, committee work, or academic pursuits that would make more frequent rounding unattractive. Physicians-in-training in teaching hospitals have educational requirements and dutyhour restrictions that may limit their ability to round more frequently. ${ }^{29,30}$ There is also a need to define reasonable minimum standards for discharge processes (e.g., provision of patient education, discharge medications, and paperwork) for short-stay patients and those requiring prolonged and/or complex hospitalizations. Streamlined discharge processes for short-stay patients with simple illnesses may result in more efficient discharges. More efficient hospital discharges at flexible hours that are acceptable to families may require a culture shift among hospital staff. Although the palatability of off-hours inpatient discharges has not been explored with patients and families, some may prefer this approach.

\section{Limitations}

There are several limitations to our study. First, the time of discharge is recorded differently for the ED and inpatient settings in our administrative dataset as a function of the electronic medical record systems in these 2 environments. Discharge from the ED reflects that time the patient left the ED but discharge from the inpatient setting reflects the time the patient exits the hospital ADT system. This likely biases our results toward longer LOS in the inpatient setting. We expect this would shift the band of inpatient discharges later in the day but do not expect this to alter the observed difference in discharge patterns between the ED and inpatient settings.

Second, administrative data do not provide information about the physicians making the discharge decisions or valid reasons a child would remain in the hospital after they improved clinically including time for teaching or care coordination. Our results therefore overestimate the amount of "excess time" associated with inpatient care. Future research is needed to determine the actual duration of time between when a child is clinically well enough for discharge home and when a child is actually discharged. There is also a need to understand discharge decision making and to identify the nonclinical factors that contribute to discharge delays.

Third, our data sample was taken from visits made by children to a pediatric ED nested within a general ED where children are admitted to an adjoining tertiary care academic children's hospital. Our results may not be generalizable to other settings in which children receive hospital-based care, including freestanding children's hospitals and general hospitals that admit children.

Fourth, we focused our analyses on children with respiratory conditions, excluding visits with diagnoses suggestive of complex comorbid conditions and severe illnesses. Although we anticipate that children with these respiratory conditions treated in other hospitals will be similar to our population, our results may not hold for other conditions or across the full spectrum of severity for acute respiratory illness treated within EDs and inpatient units.

Finally, our simulation model makes assumptions, such as a fixed treatment times and discharge process length, which do not capture the clinical nuances of an individual child's response to hospitalbased treatment. Though much of the clinical complexity of hospital operations is not taken into account in the model, our intended purpose, to explore the influence of different rounding times on LOS, remains valid. 


\section{CONCLUSIONS}

For children obtaining emergency care for respiratory illnesses, discharges from the ED occur around the clock, whereas discharges from the inpatient general care unit are concentrated during afternoon hours. Simulation models illustrate the potential to reduce hospital LOS by adding rounding sessions. Extending the hours of discharge for hospitalized children with respiratory illnesses may increase efficiency of care but could result in unintended consequences such as fewer opportunities for patient education.

\section{Acknowledgments}

The authors acknowledge the Center for Healthcare Engineering and Patient Safety, the Bonder Foundation, the Doctors Company Foundation, the Center for Research on Learning and Teaching, and the University of Michigan Summer Undergraduate Research in Engineering Program for their support of the students who contributed to this project.

Disclosures: Funding for data analysis for this project was supported by a grant from the Center for Healthcare Research and Transformation (Dr. Macy). Support for the students working on this project (East, Burns, O'Gara, and Card) was provided through grants from the Center for Research and Teaching at the University of Michigan, the Center for Healthcare Engineering and Patient Safety at the University of Michigan, the Seth Bonder Foundation, and the Doctors Company Foundation. The authors have no financial or other conflicts of interest to disclose.

\section{References}

1. Fieldston ES, Hall M, Sills MR, et al. Children's hospitals do not acutely respond to high occupancy. Pediatrics. 2010;125(5):974-981.

2. Fieldston ES, Hall M, Shah SS, et al. Addressing inpatient crowding by smoothing occupancy at children's hospitals. J Hosp Med. 2011; 6(8):462-468.

3. Schuur JD, Venkatesh AK. The growing role of emergency departments in hospital admissions. N Engl J Med. 2012;367(5):391-393.

4. Camargo CA Jr, Rachelefsky G, Schatz M. Managing asthma exacerbations in the emergency department: summary of the National Asthma Education and Prevention Program Expert Panel Report 3 guidelines for the management of asthma exacerbations. J Allergy Clin Immunol. 2009;124(2 suppl):S5-S14.

5. American Academy of Pediatrics Subcommittee on Diagnosis and Management of Bronchiolitis. Diagnosis and management of bronchiolitis. Pediatrics. 2006;118(4):1774-1793.

6. Bradley JS, Byington CL, Shah SS, et al. Executive summary: the management of community-acquired pneumonia in infants and children older than 3 months of age: clinical practice guidelines by the Pediatric Infectious Diseases Society and the Infectious Diseases Society of America. Clin Infect Dis. 2011;53(7):617-630.

7. Russell KF, Liang Y, O'Gorman K, Johnson DW, Klassen TP. Glucocorticoids for croup. Cochrane Database Syst Rev. 2011(1): CD001955.

8. Alpern ER, Stanley RM, Gorelick MH, et al. Epidemiology of a pediatric emergency medicine research network: the PECARN Core Data Project. Pediatr Emerg Care. 2006;22(10):689-699.

9. Friedman B, Berdahl T, Simpson LA, et al. Annual report on health care for children and youth in the United States: focus on trends in hospital use and quality. Acad Pediatr. 2011;11(4):263-279.
10. Macy ML, Stanley RM, Lozon MM, Sasson C, Gebremariam A, Davis MM. Trends in high-turnover stays among children hospitalized in the United States, 1993-2003. Pediatrics. 2009;123(3):996-1002.

11. McConnochie KM, Russo MJ, McBride JT, Szilagyi PG, Brooks AM, Roghmann KJ. How commonly are children hospitalized for asthma eligible for care in alternative settings? Arch Pediatr Adolesc Med. 1999;153(1):49-55.

12. Macy ML, Stanley RM, Sasson C, Gebremariam A, Davis MM. High turnover stays for pediatric asthma in the United States: analysis of the 2006 Kids' Inpatient Database. Med Care. 2010;48(9):827-833.

13. Macy ML, Hall M, Shah SS, et al. Pediatric observation status: are we overlooking a growing population in children's hospitals? J Hosp Med. 2012;7(7):530-536.

14. Macy ML, Hall M, Shah SS, et al. Differences in designations of observation care in US freestanding children's hospitals: are they virtual or real? J Hosp Med. 2012;7(4):287-293.

15. Derlet RW, Richards JR. Overcrowding in the nation's emergency departments: complex causes and disturbing effects. Ann Emerg Med. 2000;35(1):63-68.

16. Derlet R, Richards J, Kravitz R. Frequent overcrowding in U.S. emergency departments. Acad Emerg Med. 2001;8(2):151-155.

17. Trzeciak S, Rivers EP. Emergency department overcrowding in the United States: an emerging threat to patient safety and public health. Emerg Med J. 2003;20(5):402-405.

18. Rabin E, Kocher K, McClelland M, et al. Solutions to emergency department 'boarding' [and crowding are underused and may need to be legislated. Health Aff (Millwood). 2012;31(8):1757-1766.

19. Olshaker JS. Managing emergency department overcrowding. Emerg Med Clin North Am. 2009;27(4):593-603, viii.

20. Overcrowding crisis in our nation's emergency departments: is our safety net unraveling? Pediatrics. 2004;114(3):878-888.

21. McCarthy ML, Zeger SL, Ding R, et al. Crowding delays treatment and lengthens emergency department length of stay, even among highacuity patients. Ann Emerg Med. 2009;54(4):492-503.e494.

22. Langhan TS. Do elective surgical and medical admissions impact emergency department length of stay measurements? Clin Invest Med. 2007;30(5):E177-E182.

23. Khanna S, Boyle J, Good N, Lind J. Unravelling relationships: hospital occupancy levels, discharge timing and emergency department access block. Emerg Med Australas. 2012;24(5):510-517.

24. Hillier DF, Parry GJ, Shannon MW, Stack AM. The effect of hospital bed occupancy on throughput in the pediatric emergency department. Ann Emerg Med. 2009;53(6):767-776.e763.

25. Lorch SA, Millman AM, Zhang X, Even-Shoshan O, Silber JH. Impact of admission-day crowding on the length of stay of pediatric hospitalizations. Pediatrics. 2008;121(4):e718-e730.

26. Powell ES, Khare RK, Venkatesh AK, Van Roo BD, Adams JG, Reinhardt G. The relationship between inpatient discharge timing and emergency department boarding. J Emerg Med. 2012;42(2):186-196.

27. Kolb EMW, Taesik L, Peck J. Effect of coupling between emergency department and inpatient unit on the overcrowding in emergency department. Paper presented at: Winter Simulation Conference; 2007; Washington, DC.

28. Iantorno S, Fieldston E. Hospitals are not hotels: high-quality discharges occur around the clock. JAMA Pediatr. 2013;167(7):596597.

29. Antiel RM, Thompson SM, Hafferty FW, et al. Duty hour recommendations and implications for meeting the ACGME core competencies: views of residency directors. Mayo Clin Proc. 2011;86(3):185-191.

30. Auger KA, Sieplinga KR, Simmons JM, Gonzalez Del Rey JA. Failure to thrive: pediatric residents weigh in on feasibility trial of the proposed 2008 institute of medicine work hour restrictions. J Grad Med Educ. 2009;1(2):181-184. 